\title{
Functions of Baire Class One over a Bishop Topology
}

\author{
Iosif Petrakis $^{(\otimes)}$ (iD \\ University of Munich, Munich, Germany \\ petrakis@math.lmu.de
}

\begin{abstract}
If $\mathcal{T}$ is a topology of open sets on a set $X$, a real-valued function on $X$ is of Baire class one over $\mathcal{T}$, if it is the pointwise limit of a sequence of functions in the corresponding ring of continuous functions $C(X)$. If $F$ is a Bishop topology of functions on $X$, a constructive and function-theoretic alternative to $\mathcal{T}$ introduced by Bishop, we define a real-valued function on $X$ to be of Baire class one over $F$, if it is the pointwise limit of a sequence of functions in $F$. We show that the set $B_{1}(F)$ of functions of Baire class one over a given Bishop topology $F$ on a set $X$ is a Bishop topology on $X$. Consequently, notions and results from the general theory of Bishop spaces are naturally translated to the study of Baire class one-functions. We work within Bishop's informal system of constructive mathematics $\mathrm{BISH}^{*}$, that is BISH extended with inductive definitions with rules of countably many premises.
\end{abstract}

\section{Introduction}

If $\mathcal{T}$ is a topology of open sets on a set $X$, a function $f: X \rightarrow \mathbb{R}$ is of Baire class one over $\mathcal{T}$, if it is the pointwise limit of a sequence of functions in the corresponding ring of continuous functions $C(X)$. Such functions, which may no longer be in $C(X)$, were introduced by Baire in [2], suggesting the use of functions, instead of sets, to tackle problems of real analysis. If $B_{0}(X)=C(X)$, and if $B_{1}(X)$ is the set of all Baire class one-functions, one defines for every ordinal $\alpha \leq \Omega$, where $\Omega$ is the first uncountable ordinal $\Omega$, the set

$$
B_{\alpha}(X):=\operatorname{Lim}_{p}\left(\bigcup_{\beta<\alpha} B_{\beta}(X)\right),
$$

where, if $\mathbb{F}(X)$ is the set of real-valued functions on $X, \Phi \subseteq \mathbb{F}(X)$, and $f_{n} \stackrel{p}{\longrightarrow} f$ denotes that $f$ is the pointwise limit of $\left(f_{n}\right)_{n=1}^{\infty}$, we set

$$
\operatorname{Lim}_{p}(\Phi):=\left\{f \in \mathbb{F}(X) \mid \exists_{\left(f_{n}\right)_{n=1}^{\infty} \subseteq \Phi}\left(f_{n} \stackrel{p}{\longrightarrow} f\right)\right\} .
$$

The theory of Baire class-functions is a function-theoretic version of the theory of Baire sets i.e., of sets the characteristic function of which is in some Baire class ${ }^{1}$.

\footnotetext{
${ }^{1}$ For that see the Lebesgue-Hausdorff theorem in [15], p. 393, and Lorch's comment in [16], p. 751, on the "coextension" of the two theories.
} 
Generalisations of Baire class functions between metrizable spaces are central objects of study in descriptive set theory (see e.g., $[13,14]$ ), with Baire class one-functions having applications to the theory of Banach spaces (see e.g., [9]).

The theory of Bishop spaces (TBS) is a function-theoretic approach to constructive topology within Bishop's informal system of constructive mathematics BISH. The fundamental notion of a function space, here called a Bishop space, was only introduced by Bishop in [3], p. 71. The subject was revived much later by Bridges in [5], where the notion of a Bishop morphism was also defined, and by Ishihara in [11]. In [18-27] we try to develop TBS.

A Bishop topology of functions $F$ on a set $X$ is a set of real-valued functions defined on $X$ that satisfies the main properties of the set of all Bishop continuous functions from $\mathbb{R}$ to $\mathbb{R}$. A function $\phi: \mathbb{R} \rightarrow \mathbb{R}$ is called (Bishop) continuous, if it is uniformly continuous on every bounded subset $B$ of $\mathbb{R}$ i.e., if for every bounded subset $^{2} B$ of $\mathbb{R}$ and for every $\varepsilon>0$ there exists $\omega_{\phi, B}(\varepsilon)>0$ such that

$$
\forall_{a, b \in B}\left(|a-b| \leq \omega_{\phi, B}(\varepsilon) \Rightarrow|\phi(a)-\phi(b)| \leq \varepsilon\right),
$$

where the function $\omega_{\phi, B}: \mathbb{R}^{+} \rightarrow \mathbb{R}^{+}, \varepsilon \mapsto \omega_{\phi, B}(\varepsilon)$, is called a modulus of continu$i t y$ for $\phi$ on $B$. Their set is denoted by $\operatorname{Bic}(\mathbb{R})$, and two functions $\phi_{1}, \phi_{2} \in \operatorname{Bic}(\mathbb{R})$ are equal, if $\phi_{1}(a)=\phi_{2}(a)$, for every $a \in \mathbb{R}$. The restriction of this notion of continuity to a compact interval $[a, b]$ of $\mathbb{R}$ is equivalent to uniform continuity. By using this stronger notion of continuity, rather than the standard pointwise continuity, Bishop managed to avoid the use of fan theorem in the proof of the uniform continuity theorem and to remain "neutral" with respect to classical mathematics (CLASS), intuitionistic mathematics (INT), and intuitionistic computable mathematics (RUSS).

Extending our work [22], where the Baire sets over a Bishop topology $F$ are studied, here we give an introduction to the constructive theory of Baire class one-functions over a Bishop topology. In analogy to the classical concept, if $F$ is a Bishop topology on a set $X$, we define a function $f: X \rightarrow \mathbb{R}$ to be of Baire class one over $F$, if it is the pointwise limit of a sequence of functions in $F$. Our constructive translation of the fundamentals of the classical theory of Baire class one-functions (see e.g., [10]) within TBS is summarized by Theorem 1, according to which the set $B_{1}(F)$ of Baire class one-functions over $F$ is a Bishop topology on $X$ that includes $F$. As we explain in Sect. 5 , and based on the examples of Baire class one-functions included in Sect. 4 , this result offers a way to study constructively classically discontinuous functions.

We work within BISH* $^{*}$, that is BISH extended with inductive definitions with rules of countably many premises. A formal system for BISH* is Myhill's system CST $^{*}$, developed in [17], or CZF with dependence choice ${ }^{3}$ (see [6], p. 12), and some very weak form of Aczel's regular extension axiom (see [1]).

\footnotetext{
${ }^{2}$ It suffices to say that $\phi$ is uniformly continuous on every interval $[-n, n]$, and the quantification over the powerset of $\mathbb{R}$ is replaced by quantification over $\mathbb{N}$.

${ }^{3}$ Here we use the principle of dependent choice in the proof of Lemma 6 .
} 


\section{Fundamentals of Bishop Spaces}

In this section we include all definitions and facts necessary to the rest of the paper. All proofs not given given here are found in [18].

If $a, b \in \mathbb{R}$, let $a \vee b:=\max \{a, b\}$ and $a \wedge b:=\min \{a, b\}$. Hence, $|a|=a \vee(-a)$. If $f, g \in \mathbb{F}(X)$, let $f=_{\mathbb{F}(X)} g: \Leftrightarrow \forall_{x \in X}\left(f(x)=_{\mathbb{R}} f(y)\right)$, where for all definitions related to $\mathbb{R}$ see [4], chapter 2 . If $f \in \mathbb{F}(X)$ and $\left(f_{n}\right)_{n=1}^{\infty} \subseteq \mathbb{F}(X)$, the pointwise convergence $\left(f_{n}\right) \stackrel{p}{\longrightarrow} f$ and the uniform convergence $\left(f_{n}\right) \stackrel{u}{\longrightarrow} f$ on $A \subseteq X$ are defined, respectively, by

$$
\begin{gathered}
\left(f_{n}\right) \stackrel{p}{\longrightarrow} f: \Leftrightarrow \forall_{x \in A} \forall_{\epsilon>0} \exists_{n_{0} \in \mathbb{N}} \forall_{n \geq n_{0}}\left(\left|f_{n}(x)-f(x)\right|<\varepsilon\right), \\
\left(f_{n}\right) \stackrel{u}{\longrightarrow} f: \Leftrightarrow \forall_{\epsilon>0} \exists_{n_{0} \in \mathbb{N}} \forall_{n \geq n_{0}}\left(U\left(A ; f, f_{n}, \varepsilon\right)\right), \\
U\left(A ; f, f_{n}, \varepsilon\right): \Leftrightarrow \forall_{x \in A}\left(\left|f_{n}(x)-f(x)\right|<\varepsilon\right) .
\end{gathered}
$$

A set $X$ is inhabited, if it has an element. We denote by $\bar{a}$, or simply by $a$, the constant function on $X$ with value $a \in \mathbb{R}$, and by $\operatorname{Const}(X)$ their set.

Definition 1. A Bishop space is a pair $\mathcal{F}:=(X, F)$, where $X$ is an inhabited set and $F$ is an extensional subset of $\mathbb{F}(X)$ i.e., $[f \in F \& g=\mathbb{F}(X) f] \Rightarrow g \in F$, such that the following conditions hold:

$\left(\mathrm{BS}_{1}\right) \operatorname{Const}(X) \subseteq F$.

$\left(\mathrm{BS}_{2}\right)$ If $f, g \in F$, then $f+g \in F$.

$\left(\mathrm{BS}_{3}\right)$ If $f \in F$ and $\phi \in \operatorname{Bic}(\mathbb{R})$, then $\phi \circ f \in F$.

$\left(\mathrm{BS}_{4}\right)$ If $f \in \mathbb{F}(X)$ and $\left(f_{n}\right)_{n=1}^{\infty} \subseteq F$ such that $\left(f_{n}\right) \stackrel{u}{\longrightarrow} f$ on $X$, then $f \in F$.

We call $F$ a Bishop topology on $X$. If $\mathcal{G}:=(Y, G)$ is a Bishop space, a Bishop morphism from $\mathcal{F}$ to $\mathcal{G}$ is a function $h: X \rightarrow Y$ such that $\forall_{g \in G}(g \circ h \in F)$. We denote by $\operatorname{Mor}(\mathcal{F}, \mathcal{G})$ the set of Bishop morphisms from $\mathcal{F}$ to $\mathcal{G}$. If $h \in \operatorname{Mor}(\mathcal{F}, \mathcal{G})$, we say that $h$ is open, if $\forall_{f \in F} \exists_{g \in G}(f=g \circ h)$.

A Bishop morphism $h \in \operatorname{Mor}(\mathcal{F}, \mathcal{G})$ is a "continuous" function from $\mathcal{F}$ to $\mathcal{G}$. If $h \in \operatorname{Mor}(\mathcal{F}, \mathcal{G})$ is a bijection, then $h^{-1} \in \operatorname{Mor}(\mathcal{G}, \mathcal{F})$ i.e., $h$ is a Bishop isomorphism, if and only if $h$ is open. Let $\mathcal{R}$ be the Bishop space of reals $(\mathbb{R}, \operatorname{Bic}(\mathbb{R}))$. It is easy to show that if $F$ is a topology on $X$, then $F=\operatorname{Mor}(\mathcal{F}, \mathcal{R})$ i.e., an element of $F$ is a real-valued "continuous" function on $X$. A Bishop topology $F$ on $X$ is an algebra and a lattice, where $f \vee g$ and $f \wedge g$ are defined pointwise, and Const $(X) \subseteq F \subseteq \mathbb{F}(X)$. If $\mathbb{F}^{*}(X)$ denotes the bounded elements of $\mathbb{F}(X)$, then $F^{*}:=F \cap \mathbb{F}^{*}(X)$ is a Bishop topology on $X$. If $x={ }_{X} y$ is the given equality on $X$, a Bishop topology $F$ on $X$ separates the points of $X$, or $F$ is completely regular (see [19] for their importance in the theory of Bishop spaces), if

$$
\forall_{x, y \in X}\left[\forall_{f \in F}\left(f(x)=_{\mathbb{R}} f(y)\right) \Rightarrow x==_{X} y\right] .
$$

In Proposition 5.1.3. of [18] it is shown that $F$ separates the points of $X$ if and only if the induced by $F$ apartness relation on $X$

$$
x \neq_{F} y: \Leftrightarrow \exists_{f \in F}\left(f(x) \neq_{\mathbb{R}} f(y)\right)
$$


is tight i.e., $\neg\left(x \neq_{F} y\right) \Rightarrow x=_{X} \quad y$. We use the last result in the proof of Proposition 1(iv). An apartness relation on $X$ is a positively defined inequality on $X$. E.g., if $a, b \in \mathbb{R}$, then $a \neq \neq_{\mathbb{R}} b: \Leftrightarrow|a-b|>0$. In Proposition 5.1.2. of [18] we show that $a \neq_{\mathbb{R}} b \Leftrightarrow a \neq_{\operatorname{Bic}(\mathbb{R})} b$.

Definition 2. Turning the definitional clauses $\left(\mathrm{BS}_{1}\right)-\left(\mathrm{BS}_{4}\right)$ into inductive rules, the least topology $\bigvee F_{0}$ generated by a set $F_{0} \subseteq \mathbb{F}(X)$, called a subbase of $\bigvee F_{0}$, is defined by the following inductive rules:

$$
\begin{gathered}
\frac{f_{0} \in F_{0}}{f_{0} \in \bigvee F_{0}}, \quad \frac{f \in \bigvee F_{0}, g \in \mathbb{F}(X), g=\mathbb{F}(X) f}{g \in \bigvee F_{0}}, \quad \frac{a \in \mathbb{R}}{\bar{a} \in \bigvee F_{0}}, \quad \frac{f, g \in \bigvee F_{0}}{f+g \in \bigvee F_{0}}, \\
\frac{f \in \bigvee F_{0}, \phi \in \operatorname{Bic}(\mathbb{R})}{\phi \circ f \in \bigvee F_{0}}, \quad \frac{f \in \mathbb{F}(X),\left(g \in \bigvee F_{0}, \mathrm{U}(X ; f, g, \varepsilon)\right)_{\epsilon>0}}{f \in \bigvee F_{0}},
\end{gathered}
$$

where the last rule is reduced to the following rule with countably many premisses

$$
\frac{f \in \mathbb{F}(X), g_{1} \in \bigvee F_{0}, \mathrm{U}\left(X ; f, g_{1}, \frac{1}{2}\right), g_{2} \in \bigvee F_{0}, \mathrm{U}\left(X ; f, g_{2}, \frac{1}{4}\right), \ldots}{f \in \bigvee F_{0}} .
$$

The above rules induce the corresponding induction principle Ind $_{\bigvee} F_{0}$ on $\bigvee F_{0}$. If $A \subseteq X$, the relative topology $F_{\mid A}$ on $A$ has the set $\left\{f_{\mid A} \mid f \in F\right\}$ as a subbase. Unless otherwise stated, from now on, $X, Y$ are inhabited sets, and $F, G$ are Bishop topologies on $X$ and $Y$, respectively.

\section{The Bishop Topology of Baire Class One-Functions}

Definition 3. A function $g \in \mathbb{F}(X)$ is called of Baire class one over $F$, or simply of Baire class one when $F$ is clear from the context, if there is a sequence $\left(f_{n}\right)_{n=1}^{\infty} \subseteq F$ such that $\left(f_{n}\right) \stackrel{p}{\longrightarrow} g$ on $X$. We denote their set by $B_{1}(F)$.

Lemma 1. Let $\left(f_{n}\right)_{n=1}^{\infty} \subseteq \mathbb{F}(X)$ and $g \in \mathbb{F}(X)$ with $\left(f_{n}\right) \stackrel{p}{\longrightarrow} g$. If $x \in X$, there is $M_{x}>0$, such that $\left\{f_{n}(x) \mid n \geq 1\right\} \cup\{g(x)\} \subseteq\left[-M_{x}, M_{x}\right]$.

Proof. Let $n_{0} \geq 1$ such that if $n \geq n_{0}$, then $\left|f_{n}(x)-g(x)\right| \leq 1$, hence $\left|f_{n}(x)\right| \leq\left|f_{n}(x)-g(x)\right|+|g(x)| \leq 1+|g(x)|$. If $M_{x}:=\max \{1+$ $\left.|g(x)|,\left|f_{1}(x)\right|, \ldots,\left|f_{n_{0}-1}(x)\right|\right\}$, then $\left|f_{n}(x)\right| \leq M_{x}$, for every $n \geq 1$, and $|g(x)| \leq$ $M_{x}$.

Lemma 2. If $g \in B_{1}(F)$ and $\phi \in \operatorname{Bic}(\mathbb{R})$, then $\phi \circ g \in B_{1}(F)$.

Proof. Let $\left(f_{n}\right)_{n=1}^{\infty} \subseteq F$ such that $\left(f_{n}\right) \stackrel{p}{\longrightarrow} g$. If $x \in X$ and $\varepsilon>0$ are fixed, there is $n_{0}\left(\omega_{\phi,\left[-M_{x}, M_{x}\right]}(\varepsilon)\right)$ such that for every $n \geq n_{0}\left(\omega_{\phi,\left[-M_{x}, M_{x}\right]}(\varepsilon)\right)$ we have that $\left|f_{n}(x)-g(x)\right| \leq \omega_{\phi,\left[-M_{x}, M_{x}\right]}(\varepsilon)$. Since $f_{n}(x) \in\left[-M_{x}, M_{x}\right]$, for every $n \geq 1$, and $g(x) \in\left[-M_{x}, M_{x}\right]$, by the uniform continuity of $\phi$ we have that

$$
\left|f_{n}(x)-g(x)\right| \leq \omega_{\phi,\left[-M_{x}, M_{x}\right]}(\varepsilon) \Rightarrow \mid \phi\left(f_{n}(x)\right)-\phi(g(x) \mid \leq \varepsilon .
$$

Hence, for every $n \geq m_{0}(\varepsilon):=n_{0}\left(\omega_{\phi,\left[-M_{x}, M_{x}\right]}(\varepsilon)\right)$ we have that $\mid\left(\phi \circ f_{n}\right)(x)-$ $(\phi \circ g)(x) \mid \leq \varepsilon$. Since $\varepsilon>0$ is arbitrary, we get $\left(\phi \circ f_{n}\right)(x) \stackrel{n}{\longrightarrow}(\phi \circ g)(x)$. Since $x \in X$ is arbitrary, we get $\phi \circ f_{n} \stackrel{p}{\longrightarrow} \phi \circ g$. 
Note that $(\mathbb{R}, \vee, \wedge)$ is not a distributive lattice, since not even $(\mathbb{Q}, \vee, \wedge)$ is one. For the properties of $a \wedge b$ and $a \vee c$ used in the next proof see [7], p. 52.

Lemma 3. If $a, b, c, M \in \mathbb{R}$ and $M>0$, the following hold.

(i) If $a \leq b$, then $a \vee c \leq b \vee c$ and $a \wedge c \leq b \wedge c$.

(ii) $[a \vee(-M)] \wedge M=[a \wedge M] \vee(-M)$.

Proof. (i) Since $b \leq b \vee c$, we get $a \leq b \vee c$. Since also $c \leq b \vee c$, we get $a \vee c \leq b \vee c$. Since $a \wedge c \leq a \leq b$, and since also $a \wedge c \leq c$, we get $a \wedge c \leq b \wedge c$.

(ii) By Corollary 2.17 in [4], p. 26, $a>-M$ or $a<M$. If $a>-M$, then $[a \vee(-M)] \wedge M=a \wedge M$ and, since $a, M>-M$ we also get $a \wedge M>-M$, hence $[a \wedge M] \vee(-M)=a \wedge M$. If $a<M$, and since also $-M<M$, we get $a \vee(-M) \vee M$, hence $[a \vee(-M)] \wedge M=a \vee(-M)$. Moreover, $[a \wedge M] \vee(-M)=a \vee(-M)$ and the required equality holds.

Lemma 4. If $g \in B_{1}(F)$ is bounded by some $M>0$, there is a sequence $\left(h_{n}\right)_{n=1}^{\infty} \subseteq F$ such that $\left(h_{n}\right) \stackrel{p}{\longrightarrow} g$ and $h_{n}$ is bounded by $M$, for every $n \geq 1$.

Proof. If $\left(f_{n}\right)_{n=1}^{\infty} \subseteq F$ such that $\left(f_{n}\right) \stackrel{p}{\longrightarrow} g$, let $h_{n}:=\left[f_{n} \vee(-M)\right] \wedge M \in F$, for every $n \geq 1$. We show that $\left(h_{n}\right) \stackrel{p}{\longrightarrow} g$. Let $x \in X, \varepsilon>0$ and $n_{0}(\varepsilon) \geq 1$, such that for every $n \geq n_{0}(\varepsilon)$ we have that $\left|f_{n}(x)-g(x)\right| \leq \varepsilon$, or equivalently $g(x)-\varepsilon \leq f_{n}(x) \leq g(x)+\varepsilon$. By Lemma $3(\mathrm{i})$, and since $-M \leq g(x) \leq M$, we get

$$
f_{n}(x) \vee(-M) \leq[g(x)+\varepsilon] \vee(-M)=g(x)+\varepsilon .
$$

Hence

$$
\left[f_{n}(x) \vee(-M)\right] \wedge M \leq f_{n}(x) \vee(-M) \leq[g(x)+\varepsilon] \vee(-M)=g(x)+\varepsilon
$$

i.e., $h_{n}(x)-g(x) \leq \varepsilon$. Since $g(x)-\varepsilon \leq f_{n}(x)$, by Lemma $3(\mathrm{i})$ we get $[g(x)-\varepsilon] \wedge$ $M \leq f_{n}(x) \wedge M$. Since $g(x)-\varepsilon \leq g(x) \leq M$, we get $g(x)-\varepsilon=[g(x)-\varepsilon] \wedge M \leq$ $f_{n}(x) \wedge M$, hence by Lemma 3(ii) we get

$$
g(x)-\varepsilon \leq f_{n}(x) \wedge M \leq\left[f_{n}(x) \wedge M\right] \vee(-M)=\left[f_{n}(x) \vee(-M)\right] \wedge M
$$

i.e., $g(x)-\varepsilon \leq h_{n}(x)$, which implies $g(x)-h_{n}(x) \leq \varepsilon$. Since we have already shown that $h_{n}(x)-g(x) \leq \varepsilon$, by the definition of $\left|h_{n}(x)-g(x)\right|$ we conclude that $\left|h_{n}(x)-g(x)\right| \leq \varepsilon$, for every $n \geq n_{0}(\varepsilon)$. Of course, $\left|h_{n}\right| \leq M$, for every $n \geq 1$.

The proofs of the following two lemmas for $B_{1}(X)$ (see [8]) are constructive.

Lemma 5. Let $\left(g_{k}\right)_{k=1}^{\infty} \subseteq B_{1}(F)$ and $\left(M_{k}\right)_{k=1}^{\infty} \subseteq \mathbb{R}$ with $M_{k}>0$, for every $k \geq$ 1 , and $\sum_{k=1}^{\infty} M_{k} \in \mathbb{R}$. If $\left|g_{k}\right| \leq M_{k}$, for every $k \geq 1$, then $g=\sum_{k=1}^{\infty} g_{k} \in B_{1}(F)$.

Proof. Since $g_{k}$ is bounded by $M_{k}$, for every $k \geq 1$, by Lemma 4 there is $\left(f_{m}^{k}\right)_{m=1}^{\infty} \subseteq F$ with $f_{m}^{k} \stackrel{p}{\longrightarrow} g_{k}$ and $\left|f_{m}^{k}\right| \leq M_{k}$. If $n \geq 1$, let $h_{n}:=\sum_{k=1}^{n} f_{n}^{k}=$ $f_{n}^{1}+f_{n}^{2}+\ldots+f_{n}^{n} \in F$. Let $\varepsilon>0$. Since $\sum_{k=1}^{\infty} M_{k} \in \mathbb{R}$, there is $N \geq 1$ with 
$\sum_{k=N+1}^{\infty} M_{k} \leq \frac{\varepsilon}{3}$. If $x \in X$, there is $n_{0} \geq N$, such that for every $n \geq n_{0}$ we have that $\left|g_{k}(x)-f_{n}^{k}(x)\right| \leq \frac{\varepsilon}{3 N}$, for every $k \in\{1, \ldots, N\}$. If $n \geq n_{0}$, then

$$
\begin{aligned}
\left|g(x)-h_{n}(x)\right| & :=\left|\sum_{k=1}^{\infty} g_{k}(x)-\sum_{k=1}^{n} f_{n}^{k}\right| \\
& \leq\left|\sum_{k=1}^{n} g_{k}(x)-f_{n}^{k}\right|+\left|\sum_{k=n+1}^{\infty} g_{k}\right| \\
& \leq \sum_{k=1}^{n}\left|g_{k}(x)-f_{n}^{k}\right|+\sum_{k=n+1}^{\infty}\left|g_{k}\right| \\
& =\sum_{k=1}^{N}\left|g_{k}(x)-f_{n}^{k}\right|+\sum_{k=N+1}^{n}\left|g_{k}(x)-f_{n}^{k}\right|+\sum_{k=n+1}^{\infty}\left|g_{k}\right| \\
& \leq \sum_{k=1}^{N}\left|g_{k}(x)-f_{n}^{k}\right|+\sum_{k=N+1}^{n}\left|f_{n}^{k}\right|+\sum_{k=N+1}^{n}\left|g_{k}(x)\right|+\sum_{k=n+1}^{\infty}\left|g_{k}\right| \\
& =\sum_{k=1}^{N}\left|g_{k}(x)-f_{n}^{k}\right|+\sum_{k=N+1}^{n}\left|f_{n}^{k}\right|+\sum_{k=N+1}^{\infty}\left|g_{k}\right| \\
& \leq \sum_{k=1}^{N} \frac{\varepsilon}{3 N}+\sum_{k=N+1}^{n} M_{k}+\sum_{k=N+1}^{\infty} M_{k} \\
& \leq N\left(\frac{\varepsilon}{3 N}\right)+\frac{\varepsilon}{3}+\frac{\varepsilon}{3}=\varepsilon .
\end{aligned}
$$

Lemma 6. If $\left(g_{n}\right)_{n=1}^{\infty} \subseteq B_{1}(F)$ and $g \in \mathbb{F}(X)$ with $\left(g_{n}\right) \stackrel{u}{\longrightarrow} g$, then $g \in B_{1}(F)$.

Proof. Using dependent choice there is a subsequence $\left(g_{n_{k}}\right)_{k=1}^{\infty}$ of $\left(g_{n}\right)_{n=1}^{\infty}$ with $U\left(X ; g, g_{n_{k}}, \frac{1}{2^{k}}\right)$, for every $k \geq 1$. Let $h_{k}:=g_{n_{k+1}}-g_{n_{k}} \in B_{1}(F)$. If $x \in X$, then

$$
\left|h_{k}(x)\right| \leq\left|g_{n_{k+1}}-g(x)\right|+\left|g(x)-g_{n_{k}}(x)\right| \leq \frac{1}{2^{k+1}}+\frac{1}{2^{k}}=\frac{3}{2} \frac{1}{2^{k}}=: M_{k} .
$$

By Lemma 5 we have that $h:=\sum_{k=1}^{\infty} h_{k} \in B_{1}(F)$. Since

$$
\begin{aligned}
h(x) & =\lim _{N \rightarrow \infty} \sum_{k=1}^{N}\left(g_{n_{k+1}}(x)-g_{n_{k}}(x)\right) \\
& =\lim _{N \rightarrow \infty}\left[\left(g_{n_{2}}(x)-g_{n_{1}}(x)\right)+\ldots+\left(g_{n_{N+1}}(x)-g_{n_{N}}(x)\right)\right] \\
& =\lim _{N \rightarrow \infty}\left(g_{n_{N+1}}(x)-g_{n_{1}}(x)\right) \\
& =\lim _{N \rightarrow \infty}\left(g_{n_{N+1}}(x)\right)-g_{n_{1}}(x) \\
& =g(x)-g_{n_{1}}(x),
\end{aligned}
$$

we get $g=h+g_{n_{1}} \in B_{1}(F)$, as $B_{1}(F)$ is trivially closed under addition. 
Theorem 1. $B_{1}(F)$ is a Bishop topology on $X$ that includes $F$.

Proof. $B_{1}(F)$ is an extensional subset of $\mathbb{F}(X)$, since if $g \in \mathbb{F}(X)$ and $\left(f_{n}\right)_{n=1}^{\infty} \subseteq$ $F$ such that $\left(f_{n}\right) \stackrel{p}{\longrightarrow} g$ on $X$, then if $g^{*}=\mathbb{F}(X) g$, we also get $\left(f_{n}\right) \stackrel{p}{\longrightarrow} g^{*}$ on $X$. Clearly, $F \subseteq B_{1}(F)$, and hence Const $(X) \subseteq B_{1}(F)$. Moreover, $B_{1}(F)$ is closed under addition. By Lemma $2 B_{1}(F)$ is closed under composition with elements of $\operatorname{Bic}(\mathbb{R})$, and by Lemma $6 B_{1}(F)$ is closed under uniform limits.

By Theorem 1, if $g_{1}, g_{2} \in B_{1}(F)$, then $g_{1} \vee g_{2}, g_{1} \wedge g_{2}, g_{1} \cdot g_{2}$, and $\left|g_{1}\right|$ are in $B_{1}(F)$. These facts also follow trivially by the definition of $B_{1}(F)$. The importance of Theorem 1 though, is revealed by the use of the general theory of Bishop spaces in the proof of non-trivial properties of $B_{1}(F)$ that, consequently, depend only on the Bishop space-structure of $B_{1}(F)$.

Corollary 1. (i) $B_{1}(F)^{*}:=B_{1}(F) \cap \mathbb{F}^{*}(X)$ is a Bishop topology on $X$.

(ii) If $g \in B_{1}(F)$ such that $g \geq \bar{c}$, for some $c \in \mathbb{R}$ with $c>0$, then $\frac{1}{g} \in B_{1}(F)$.

(iii) If $g \in B_{1}(F)$ such that $g \geq \overline{0}$, then $\sqrt{g} \in B_{1}(F)$.

(iv) The collection $Z\left(B_{1}(F)\right)=\left\{\zeta(g) \mid g \in B_{1}(F)\right\}$ of zero sets of $B_{1}(F)$, where $\zeta(g):=\{x \in X \mid g(x)=0\}$, is closed under countable intersections.

(v) [Urysohn lemma for $B_{1}(F)$-zero sets] If $A, B \subseteq X$, then there is $h \in B_{1}(F)$ with $h(A)=0$ and $h(B)=1$ if and only if there are $g_{1}, g_{2} \in B_{1}(F)$, and $c>0$. such that $A \subseteq \zeta\left(g_{1}\right), B \subseteq \zeta\left(g_{2}\right)$, and $\left|g_{1}\right|+\left|g_{2}\right| \geq \bar{c}$.

(vi) [Urysohn extension theorem for $B_{1}(F)$ ] Let $Y \subseteq X$ such that $f_{\mid Y} \in G$, for every $f \in F$. If for every $A, B \subseteq Y$, whenever $A, B$ are separated by some function in $B_{1}(G)^{*}$, then $A, B$ are separated by some function in $B_{1}(F)^{*}$, then every $g^{*} \in B_{1}(G)^{*}$ is the restriction of some $f^{*} \in B_{1}(F)^{*}$.

Proof. These facts follow from the corresponding facts on general Bishop spaces. See [18], p. 41, for (i), Theorem 5.4.8. in [18] for (ii), [26] for a proof of (iii), Proposition 5.3.3.(ii) in [18] for (iv), Theorem 5.4.9. in [18] for (v), and the Urysohn extension theorem for general Bishop spaces in [20] for (vi).

Corollary 1, except from case (iii), are classically shown in [8] specifically for $B_{1}(X)$. Notice that in [20] we avoid quantification over the powerset of $Y$ in the formulation of the Urysohn extension theorem, formulating it predicatively.

Proposition 1. Let $x, y \in X$.

(i) If $g \in B_{1}(F)$ with $g(x) \neq_{\mathbb{R}} g(y)$, there is $f \in F$ such that $f(x) \neq_{\mathbb{R}} f(y)$.

(ii) $x \neq_{B_{1}(F)} y \Leftrightarrow x \neq_{F} y$.

(iii) The apartness $\neq_{B_{1}(F)}$ is tight if and only if the apartness $\neq_{F}$ is tight.

(iv) $B_{1}(F)$ separates the points of $X$ if and only if $F$ separates them.

$(v) B_{1}(F)$ separates the points of $X$ if and only if $B_{1}(F)^{*}$ separates them.

Proof. (i) Since $|g(x)-g(y)|>0$, let the well-defined function

$$
g^{*}(z):=\frac{1}{g(x)-g(y)} g(z)-g(y), \quad z \in X .
$$


$g^{*}$ is in $B_{1}(F), g^{*}(x)=1$ and $g^{*}(y)=0$. If $\left(f_{n}\right)_{n=1}^{\infty} \subseteq F$ with $\left(f_{n}\right) \stackrel{p}{\longrightarrow} g^{*}$, then

$$
\exists_{n_{0}^{x}\left(\frac{1}{2}\right) \in \mathbb{N}} \forall_{n \geq n_{0}^{x}\left(\frac{1}{2}\right)}\left(\left|f_{n}(x)-1\right|<\frac{1}{2}\right) \quad \& \quad \exists_{n_{0}^{y}\left(\frac{1}{2}\right) \in \mathbb{N}} \forall_{n \geq n_{0}^{y}\left(\frac{1}{2}\right)}\left(\left|f_{n}(y)\right|<\frac{1}{2}\right) .
$$

If $m:=\max \left\{n_{0}^{x}\left(\frac{1}{2}\right), n_{0}^{y}\left(\frac{1}{2}\right)\right\}$, then $f_{m} \in F$ with $f_{m}(x) \in\left(\frac{1}{2}, \frac{3}{2}\right)$ and $f_{m}(y) \in$ $\left(-\frac{1}{2}, \frac{1}{2}\right)$, hence $f_{m}(x) \neq_{\mathbb{R}} f_{m}(y)$.

(ii) If $x \neq_{B_{1}(F)} y$, there is $g \in B_{1}(F)$ such that $g(x) \neq_{\mathbb{R}} g(y)$. By (i) we get $x \neq_{F} y$. Conversely, if $x \neq_{F} y$, there is $f \in F$ with $f(x) \neq_{\mathbb{R}} f(y)$. Since $f$ is also in $B_{1}(F)$, we get $x \neq_{B_{1}(F)} y$.

(iii) Let $\neq_{B_{1}(F)}$ be tight. If $\neg\left(x \neq_{F} y\right)$, then by (ii) we get $\neg\left(x \neq_{B_{1}(F)} y\right)$, hence $x={ }_{X} y$. The converse implication is shown similarly.

(iv) It follows from (iii) and the result mentioned in Sect. 2 that a Bishop topology separates the points if and only if its induced apartness is tight.

(v) It follows from the general fact that $F$ separates the points if and only if $F^{*}$ separates them (see Proposition 5.7.2. in [18]).

Proposition 2. Let $\mathcal{F}_{1}:=\left(X, B_{1}(F)\right)$ and $\mathcal{G}_{1}:=\left(Y, B_{1}(G)\right)$.

(i) If $h \in \operatorname{Mor}(\mathcal{F}, \mathcal{G})$, then $h \in \operatorname{Mor}\left(\mathcal{F}_{1}, \mathcal{G}_{1}\right)$.

(ii) Let $h: X \rightarrow Y$ be a surjection with $\sigma: Y \rightarrow X$ a modulus of surjectivity ${ }^{4}$ for $h$ i.e., $h \circ \sigma=\operatorname{id}_{Y}$. If $h \in \operatorname{Mor}(\mathcal{F}, \mathcal{G})$ is open, then $h \in \operatorname{Mor}\left(\mathcal{F}_{1}, \mathcal{G}_{1}\right)$ is open.

Proof. (i) We need to show that $\forall_{g \in B_{1}(G)}\left(g \circ h \in B_{1}(F)\right)$. If we fix $g \in B_{1}(G)$, let $\left(g_{n}\right)_{n=1}^{\infty} \subseteq G$ such that $\left(g_{n}\right) \stackrel{p}{\longrightarrow} g$. Then, we get $\left(g_{n} \circ h\right) \stackrel{p}{\longrightarrow} g \circ h$. Since $h \in \operatorname{Mor}(\mathcal{F}, \mathcal{G})$, we have that $g_{n} \circ h \in F$, for every $n \geq 1$, and hence $g \circ h \in B_{1}(F)$. (ii) By case (i) $h \in \operatorname{Mor}\left(\mathcal{F}_{1}, \mathcal{G}_{1}\right)$. By Definition 1, if $\forall_{f \in F} \exists_{g \in G}(f=g \circ h)$, we prove $\forall_{f^{*} \in B_{1}(F)} \exists_{g^{*} \in B_{1}(G)}\left(f^{*}=g^{*} \circ h\right)$. Let $f^{*} \in B_{1}(F)$ and $\left(f_{n}\right)_{n=1}^{\infty} \subseteq F$ with $\left(f_{n}\right) \stackrel{p}{\longrightarrow} f^{*}$ on $X$. By the principle of countable choice (see [6], p. 12) there is $\left(g_{n}\right)_{n=1}^{\infty} \subseteq G$ such that $f_{n}=g_{n} \circ h$, for every $n \geq 1$. Let $g^{*}: Y \rightarrow \mathbb{R}$, defined by $g^{*}:=f^{*} \circ \sigma$. First we show that $g^{*} \circ h=_{\mathbb{F}(X)} f^{*}$. If $x \in X$, we show that

$$
\left(g^{*} \circ h\right)(x):=g^{*}(h(x)):=f^{*}(\sigma(h(x)))=f^{*}(x) .
$$

Since $h(\sigma(h(x))):=(h \circ \sigma)(h(x))==_{Y} \operatorname{id}_{Y}(h(x))=h(x)$, we get

$$
\left(g_{n} \circ h\right)(\sigma(h(x))):=g_{n}(h(\sigma(h(x))))=g_{n}(h(x)):=\left(g_{n} \circ h\right)(x)
$$

i.e., $f_{n}(\sigma(h(x)))=f_{n}(x)$, for every $n \geq 1$. Since $f_{n}(\sigma(h(x))) \stackrel{n}{\longrightarrow} f^{*}(\sigma(h(x)))$ and $f_{n}(x) \stackrel{n}{\longrightarrow} f^{*}(x)$, we get $f^{*}(\sigma(h(x)))=f^{*}(x)$. Since $B_{1}(F)$ is an extensional subset of $\mathbb{F}(X)$ and $g^{*} \circ h=\mathbb{F}(X) f^{*} \in B_{1}(F)$, we conclude that $g^{*} \circ h \in B_{1}(F)$ too. To prove $g^{*} \in B_{1}(G)$, we show that $\left(g_{n}\right) \stackrel{p}{\longrightarrow} g^{*}$. If $y \in Y$, then

$$
g_{n}(y)=g_{n}(h(\sigma(y))):=\left(g_{n} \circ h\right)(\sigma(y))=f_{n}(\sigma(y)) .
$$

Since $f_{n}(\sigma(y)) \stackrel{n}{\longrightarrow} f^{*}(\sigma(y)):=g^{*}(y)$, we conclude that $g_{n}(y) \stackrel{n}{\longrightarrow} g^{*}(y)$.

\footnotetext{
${ }^{4}$ We use $\sigma$ in order to avoid the general axiom of choice in the proof.
} 


\section{Examples of Functions of Baire Class One over $F$}

First we find an unbounded Baire class one-function over some Bishop topology. If $n \geq 1$, let $f_{n}:\{0\} \cup(0,1] \rightarrow \mathbb{R}$ defined by

$$
f_{n}(x):= \begin{cases}0 & , x=0 \\ \left(n^{2} x \wedge n\right) \wedge \frac{1}{x}, & x \in(0,1]\end{cases}
$$

Clearly, $f_{n} \leq n$, for every $n \geq 1$. If $0<x<\frac{1}{n}$, then $0<n^{2} x<n$ and $n<\frac{1}{x}$, hence $\left(n^{2} x \wedge n\right) \wedge \frac{1}{x}=\left(n^{2} x\right) \wedge \frac{1}{x}=n^{2} x$. If $\frac{1}{n} \leq x \leq 1$, then $n \leq n^{2} x \leq n^{2}$ and $\frac{1}{x} \leq n$, hence $\left(n^{2} x \wedge n\right) \wedge \frac{1}{x}=n \wedge \frac{1}{x}=\frac{1}{x}$. Hence,

$$
f_{n}(x)= \begin{cases}n^{2} x & , x \in\{0\} \cup\left(0, \frac{1}{n}\right) \\ \frac{1}{x} & , x \in\left[\frac{1}{n}, 1\right]\end{cases}
$$

If $F_{0}:=\left\{f_{n} \mid n \geq 1\right\}$, we consider the Bishop topology $\bigvee F_{0}$ on $X:=\{0\} \cup(0,1]$. Let the function $g:\{0\} \cup(0,1] \rightarrow \mathbb{R}$, defined by

$$
g(x):=\left\{\begin{array}{l}
0, x=0 \\
\frac{1}{x}, x \in(0,1]
\end{array}\right.
$$

Clearly, $g$ is unbounded on its domain. We show that $f_{n} \stackrel{p}{\longrightarrow} g$, hence $g \in$ $B_{1}\left(\bigvee F_{0}\right)$. If $x=0$, then $0=f_{n}(0) \stackrel{n}{\longrightarrow} g(0)=0$. Let $x \in(0,1]$. We fix some $\varepsilon>0$, and we find $n_{0} \geq 1$ such that $\frac{1}{n_{0}}<x$. Hence, if $n \geq n_{0}$, then $\frac{1}{n}<x$ too. Since then $n<n^{2} x$ and $\frac{1}{x}<n$, we have that

$$
\left|f_{n}(x)-g(x)\right|=\left|\left[\left(n^{2} x \wedge n\right) \wedge \frac{1}{x}\right]-\frac{1}{x}\right|=\mid\left[\left(n \wedge \frac{1}{x}\right]-\frac{1}{x}|=| \frac{1}{x}-\frac{1}{x} \mid=0 \leq \varepsilon .\right.
$$

A pseudo-compact Bishop topology is a topology all the elements of which are bounded functions. Since boundedness is a "liftable" property from $F_{0}$ to $F$ i.e., if every $f_{0} \in F_{0}$ is bounded, then every $f \in \bigvee F_{0}$ is bounded (see Proposition 3.4.4 in [18], p. 46), the topology $\bigvee F_{0}$ of the previous example is pseudo-compact, and hence the above construction is also an example of an unbounded Baire-class one function over a pseudo-compact Bishop topology!

It is immediate to show that $B_{1}(\mathbb{F}(X))=\mathbb{F}(X)$ and $B_{1}(\operatorname{Const}(X))=$ Const $(X)$. Next we find a Baire class one-function over some $F$ that is not in $F$. Let $Y:=[0,1) \cup\{1\}$ be equipped with the relative Bishop topology $C_{u}([0,1])_{\mid Y}=\operatorname{Bic}([0,1])_{\mid Y}$, where $C_{u}([0,1])$ is the Bishop topology of uniformly continuous functions on $[0,1]$, and $C_{u}([0,1])=\operatorname{Bic}([0,1])$, with $\operatorname{Bic}([0,1])$ being defined similarly to $\operatorname{Bic}(\mathbb{R})$. Let $f_{n}: Y \rightarrow \mathbb{R}$, where $f_{n}:=\mathrm{id}_{\mid Y}^{n}$, for every $n \geq 1$. By the definition of relative Bishop topology (see Definition 2) we have that $f_{n} \in \operatorname{Bic}([0,1])_{\mid Y}$, for every $n \geq 1$, and $\left(f_{n}\right) \stackrel{p}{\longrightarrow} g$, where $g: Y \rightarrow \mathbb{R}$ is given by

$$
g(x):=\left\{\begin{array}{l}
0, x \in[0,1) \\
1, x \in\{1\}
\end{array}\right.
$$


Since $Y$ is dense in $[0,1], g$ is not in $\operatorname{Bic}([0,1])_{\mid Y}$; if it was, by Proposition 4.7.15. in [18] we get $g=h_{\mid Y}$ with $h \in C_{u}([0,1])$, which is impossible.

A similar example is the following. Let $Z:=(-\infty, 1) \cup\{1\} \cup(1,+\infty)$ be equipped with the relative topology $\operatorname{Bic}(\mathbb{R})_{\mid Z}$. If $n \geq 1$, let $\phi_{n}=n \operatorname{id}_{\mathbb{R}}+(1-n) \in$ $\operatorname{Bic}(\mathbb{R})$ and $\theta_{n}:=-n \operatorname{id}_{\mathbb{R}}+(1+n) \in \operatorname{Bic}(\mathbb{R})$. If $\psi_{n}:=\left(\phi_{n} \vee 0\right) \wedge\left(\theta_{n} \vee 0\right) \in \operatorname{Bic}(\mathbb{R})$,

$$
\psi_{n}(x):= \begin{cases}0 & , x<\frac{n-1}{n} \\ n x+1-n & , x \in\left[\frac{n-1}{n}, 1\right] \\ -n x+1+n & , x \in\left(1, \frac{n+1}{n}\right] \\ 0 & , x>\frac{n+1}{n} .\end{cases}
$$

Let $\psi_{n}^{*}$ be the restriction of $\psi_{n}$ to $Z$, for every $n \geq 1$. Clearly, $\psi_{n}^{*} \stackrel{p}{\longrightarrow} h$, where

$$
h(x):=\left\{\begin{array}{l}
0, x \in(-\infty, 1) \\
1, x \in\{1\} \\
0, x \in(1+\infty)
\end{array}\right.
$$

Since $Z$ is dense in $\mathbb{R}$ (see Lemma 2.2.8. of [18]), and arguing as in the previous example, $h$ cannot be in the specified Bishop topology on $Z$.

As in the classical case, all derivatives of differentiable functions in $\mathbb{F}(\mathbb{R})$ are Baire class one-functions over $\operatorname{Bic}(\mathbb{R})$. We reformulate the definition in [4], p. 44, as follows.

Definition 4. Let $a<b, f, f^{\prime}:[a, b] \rightarrow \mathbb{R}$ (uniformly) continuous on $[a, b]$, and $\delta_{f,[a, b]}: \mathbb{R}^{+} \rightarrow \mathbb{R}^{+}$. We say that $f$ is differentiable on $[a, b]$ with derivative $f^{\prime}$ and modulus of differentiability $\delta_{f,[a, b]}$, in symbols $\operatorname{Dif}\left(f, f^{\prime}, \delta_{f,[a, b]}\right)$, if

$$
\forall_{\varepsilon>0} \forall_{x, y \in[a, b]}\left(|y-x| \leq \delta_{f,[a, b]}(\varepsilon) \Rightarrow\left|f(y)-f(x)-f^{\prime}(x)(y-x)\right| \leq \varepsilon|y-x|\right) .
$$

If $\phi, \phi^{\prime} \in \operatorname{Bic}(\mathbb{R})$, we say that $\phi$ is differentiable with derivative $\phi^{\prime}$, in symbols $\operatorname{Dif}\left(\phi, \phi^{\prime}\right)$, if for every $n \geq 1$ we have that $\operatorname{Dif}\left(\phi_{\mid[-n, n]}, \phi_{\mid[-n, n]}^{\prime}, \delta_{\phi_{\mid[-n, n]},[-n, n]}\right)$.

Proposition 3. If $\phi, \phi^{\prime} \in \operatorname{Bic}(\mathbb{R})$ such that $\operatorname{Dif}\left(\phi, \phi^{\prime}\right)$, then $\phi^{\prime} \in B_{1}(\operatorname{Bic}(\mathbb{R}))$.

Proof. If $n \geq 1$, let $\phi_{n}:=n\left[\phi \circ\left(\operatorname{id}_{\mathbb{R}}+\frac{1}{n}\right)-\phi\right] \in \operatorname{Bic}(\mathbb{R})$. We show that $\left(\phi_{n}\right) \stackrel{p}{\longrightarrow} \phi^{\prime}$. Let $x \in \mathbb{R}$ and $\varepsilon>0$. Let $N \geq 1$ with $x \in[-N, N]$. Since $\left(x+\frac{1}{n}\right) \stackrel{n}{\longrightarrow} x$ and $\delta_{\phi,[-N, N]}(\varepsilon)>0$, there is $n_{0} \geq 1$ such that for every $n \geq n_{0}$ we have that $x+\frac{1}{n} \in[-N, N]$ and $\frac{1}{n} \leq \delta_{\phi,[-N, N]}(\varepsilon)$, hence $\frac{1}{n}=\mid\left(x+\frac{1}{n}-x \mid \leq \delta_{\phi,[-N, N]}(\varepsilon)\right.$, and by Definition 4 we have that

$$
\begin{aligned}
& \left|\phi\left(x+\frac{1}{n}\right)-\phi(x)-\phi^{\prime}(x)\left(x+\frac{1}{n}-x\right)\right| \leq \varepsilon\left|x+\frac{1}{n}-x\right| \Leftrightarrow \\
& \left|\phi\left(x+\frac{1}{n}\right)-\phi(x)-\phi^{\prime}(x) \frac{1}{n}\right| \leq \varepsilon \frac{1}{n} \Rightarrow \\
& \left|n \phi\left(x+\frac{1}{n}\right)-n \phi(x)-\phi^{\prime}(x)\right| \leq \varepsilon \Leftrightarrow \\
& \left|\phi_{n}(x)-\phi^{\prime}(x)\right| \leq \varepsilon .
\end{aligned}
$$




\section{Concluding Comments}

In this paper we introduced the notion of a function of Baire class one over a Bishop topology $F$, translating a fundamental notion of classical real analysis and topology into the constructive topology of Bishop spaces. Our central result, that the set $B_{1}(F)$ of Baire class one-functions over $F$ is a Bishop topology that includes $F$, is used to apply concepts and results from the general theory of Bishop spaces to the theory of functions of Baire class one over a Bishop topology. These first applications suggest that the structure of Bishop space, treated classically, would also be useful to the classical study of function spaces like $B_{1}(X)$.

For constructive topology, the fact that $B_{1}(F)$ is a Bishop topology provides a second way, within the theory of Bishop spaces, to treat classically discontinuous, real-valued functions as "continuous" i.e., as Bishop morphisms. The first way is to consider such discontinuous functions as elements of a subbase $F_{0}$. Since by definition $F_{0} \subseteq \bigvee F_{0}$, the elements of $F_{0}$ are Bishop morphisms from the resulting least Bishop space $\mathcal{F}$ to the Bishop space $\mathcal{R}$ of reals. In [27], and based on a notion of convergence of test functions introduced by Ishihara, we follow this way to make the Dirac delta function $\delta$ and the Heaviside step function $H$ "continuous". We consider a certain set $D_{0}(\mathbb{R})$ of linear maps on the test functions on $\mathbb{R}$, where $\delta, H \in D_{0}(\mathbb{R})$, and the Bishop topology $\bigvee D_{0}(\mathbb{R})$ is used to define the set of distributions on $\mathbb{R}$. The second way, is to start from a Bishop topology $F$ and find elements of $B_{1}(F)$ i.e., Bishop morphisms from $\mathcal{F}_{1}$ to $\mathcal{R}$, that are pointwise discontinuous, as the functions $g$ and $h$ in the last two example before Definition 4. This second way is sort of a constructive analogue to the classical result that the points of pointwise continuity of some $f \in B_{1}(\mathbb{R})$ is dense in $\mathbb{R}$, hence $f$ is almost everywhere continuous.

There are numerous interesting questions stemming from this introductory work. Can we prove constructively that the characteristic function of a (complemented) Baire set $\boldsymbol{B}=\left(B^{1}, B^{0}\right)$ over a Bishop topology $F$ (see [22]) is a Baire class-one function over the relative topology $F_{\mid B^{1} \cup B^{0}}$ ? Can we show constructively other classical characterisations of $B_{1}(X)$, like for example through $F_{\sigma}$-sets? What is the exact relation between $B_{1}(F)_{\mid A}$ and $B_{1}\left(F_{\mid A}\right)$, or between $B_{1}(F \times G)$ and the product Bishop topology (see [18], Sect. 4.1 for its definition) $B_{1}(F) \times B_{1}(G)$ ? How far can we go constructively with the study of Baire class two-functions?

A base of a Bishop topology $F$ is a subset $B$ of $F$ such that every $f \in F$ is the uniform limit of a sequence in $B$. If $B$ is a base of $F$, it follows easily that

$$
\operatorname{Lim}_{p}(B)=B_{1}(F),
$$

hence for the uniform closure $\overline{\operatorname{Lim}_{p}(B)}$ of $B$ in $\mathbb{F}(X)$ we get

$$
\overline{\operatorname{Lim}_{p}(B)}=\overline{B_{1}(F)}=B_{1}(F)
$$

i.e., $\operatorname{Lim}_{p}(B)$ is a base of $B_{1}(F)$. If $F_{0} \subseteq F$ is a subbase of $F$ i.e., $F=\bigvee F_{0}$, we have that $\operatorname{Lim}_{p}\left(F_{0}\right) \subseteq \operatorname{Lim}_{p}\left(\bigvee F_{0}\right)=B_{1}(F)$, hence $\bigvee \operatorname{Lim}_{p}\left(F_{0}\right) \subseteq B_{1}(F)$. When does the inverse inclusion also hold? 
We hope to address some of these questions in a future work.

\section{References}

1. Aczel, P., Rathjen, M.: Constructive Set Theory, Book Draft (2010)

2. Baire, R.-L.: Sur les fonctions des variable réelles. Annali. Mat. 3, 1-123 (1899)

3. Bishop, E.: Foundations of Constructive Analysis. McGraw-Hill, New York City (1967)

4. Bishop, E., Bridges, D.S.: Constructive Analysis, Grundlehren der math. Wissenschaften, vol. 279, Springer, Heidelberg (1985)

5. Bridges, D.S.: Reflections on function spaces. Ann. Pure Appl Log. 163, 101-110 (2012)

6. Bridges, D.S., Richman, F.: Varieties of Constructive Mathematics. Cambridge University Press, Cambridge (1987)

7. Bridges, D.S., Vîţă, L.S.: Techniques of Constructive Analysis. Universitext, Springer, New York (2006)

8. Deb Ray, A., Mondal, A.: On rings of Baire one functions. Appl. Gen. Topol. 20(1), 237-249 (2019)

9. Haydon, R., Odell, E., Rosenthal, H.P.: Certain subsclasses of Baire-1 functions with Banach space applications. In: Longhorn Notes, University of Texas at Austin Functional Analysis Seminar (1987-1989)

10. $\mathrm{Hu}, \mathrm{J} .:$ Baire one functions, preprint

11. Ishihara, H.: Relating Bishop's function spaces to neighborhood spaces. Ann. Pure Appl. Log. 164, 482-490 (2013)

12. Jayne, J.E.: Space of Baire functions. I, Annales de l'institut Fourier, pp. 47-76 (1974)

13. Kechris, A.S., Louveau, A.: A classification of Baire class 1 functions. Trans. Am. Math. Soc. 318(1), 209-236 (1990)

14. Kechris, A.S.: Classical Descriptive Set Theory. Springer, Heidelberg (1995)

15. Kuratowski, K.: Topology, vol. I. Academic Press, New York (1966)

16. Lorch, E.R.: Continuity and Baire functions. Am. Math. Monthly 78(7), 748-762 (1971)

17. Myhill, J.: Constructive set theory. J. Symb. Log. 40, 347-382 (1975)

18. Petrakis, I.: Constructive Topology of Bishop Spaces, Ph.D. Thesis, LudwigMaximilians-Universität, München (2015)

19. Petrakis, I.: Completely regular Bishop spaces. In: Beckmann, A., Mitrana, V., Soskova, M. (eds.) CiE 2015. LNCS, vol. 9136, pp. 302-312. Springer, Cham (2015). https://doi.org/10.1007/978-3-319-20028-6_31

20. Petrakis, I.: The Urysohn extension theorem for Bishop spaces. In: Artemov, S., Nerode, A. (eds.) LFCS 2016. LNCS, vol. 9537, pp. 299-316. Springer, Cham (2016). https://doi.org/10.1007/978-3-319-27683-0_21

21. Petrakis, I.: A constructive function-theoretic approach to topological compactness. In: LICS, pp. 605-614 (2016)

22. Petrakis, I.: Borel and Baire sets in Bishop spaces. In: Manea, F., Martin, B., Paulusma, D., Primiero, G. (eds.) CiE 2019. LNCS, vol. 11558, pp. 240-252. Springer, Cham (2019). https://doi.org/10.1007/978-3-030-22996-2_21

23. Petrakis, I.: Constructive uniformities of pseudometrics and Bishop topologies. J. Log. Anal. 11(FT2), 1-44 (2019)

24. Petrakis, I.: Direct spectra of Bishop spaces and their limits. https://arxiv.org/ abs/1907.03273 (2019) 
25. Petrakis, I.: Embeddings of Bishop spaces. J. Logi. Comput. 30(1), 349-379 (2020). $10.1093 / \log c o m /$ exaa015

26. Petrakis, I.: A constructive theory of $C^{*}(X)$, preprint (2020)

27. Petrakis, I.: Towards a constructive approach to the theory of distributions, preprint (2020) 